\title{
A review of pesticide residue levels and their related health exposure risks
}

\author{
A. Fothergill \& A. Abdelghani \\ Tulane University School of Public Health and Tropical Medicine, USA
}

\begin{abstract}
Pesticides are used very regularly in agriculture and contribute to significantly higher crop yields. Applied prior or during the growing process and post-harvest, pesticide residues left on consumable food products might pose a potential health risk. One class of pesticides, fumigants, can be applied directly to food products post-harvest. Changes in types and amounts of pesticides used both agriculturally and post-harvest result in the need for residue analysis and health risk characterization. A number of studies have examined pesticide residues in certain food groups. Monitoring programs and/or studies in the United States, Poland, China, and others have found levels of pesticides to pose no serious health risk. A study in Croatia however, has found potentially hazardous levels of pesticides, particularly with regard to special populations. Most studies focus on individual pesticides and associated health effects, with few studies encompassing entire dietary intake of pesticide residuals. This literature review provides a summary of recent pesticide residue studies and health implications. Key words: pesticide residue, food consumption, health risk.
\end{abstract}

\section{Introduction}

The yield of crops is greatly enhanced by the use of pesticides, whether it is prior to production or as post-harvest treatment [1]. The presence of pesticide residues in foods has always been a concern, particularly fruits and vegetables consumed fresh [2]. Exposure to pesticide residues through the diet is assumed to be up to five times the magnitude of exposure through other routes such as air and drinking water [2, 3].

Pesticides can be classified by their use and chemical structure. The six main categories based on use are: insecticides, fungicides, herbicides, rodenticides, 
fumigants and insect repellents [4]. There are four main groups of insecticides based on chemical functions: organophosphates, pyrethroids, carbamates, and organochlorines [5]. Organophosphates are man-made chemicals that work by damaging the enzyme acetylcholinesterase [6], one organophosphate of particular importance in this review is chlorpyrifos, which was isolated in a number of studies. Pyrethroids are a synthetic version of the natural pesticide pyrethrin [5], and include the pesticides cypermethrin and permethrin, which were both detected in a number of the studies included here [7]. Carbamate pesticides also work by disrupting enzymes in the nervous system [5] and some carbamates (carbaryl and carbofuran) were found to be present in studies summarized here. Organochlorines have mostly been removed from the market due to their environmental effects and persistence [5]; however one study did find organochlorine (dicofol) residues [8].

Pesticides are known to be a public health issue and pesticides used in agriculture should have established residue limits and be frequently monitored. Typically, surveillance of pesticides focuses on proper use and compliance with Maximum Residue Limits (MRL). Maximum residue levels are based on the proper application of pesticides according to good agricultural practices in controlled field experiments. In order to evaluate food safety the observed levels of pesticides on foods need to be compared to health safety limits or toxicological endpoint values such as the Acceptable Daily Intake (ADI) and the Acute Reference Dose (ARfD), and consumption patterns of different foods [2]. $\mathrm{ADI}$ and ARfD are measures for the chronic and acute toxicity of a pesticide. They are usually based on animal studies with applied safety factors to account for differences in animals and humans, and quality of data. [9] Residue levels present at the time of consumer contact can depend on a variety of factors, such as application concentrations, repeated applications, and the time between pesticide application and harvest $[1,8]$. The exposure to or consumption of a particular pesticide below the health safety limit is considered "safe" but this concentration may be above the established MRL [2].

Pesticides have been linked with a wide variety of health effects, ranging from headaches and nausea to cancer $[1,10]$. Long term exposure to pesticides is a known health risk and it is increasingly being linked to cancer, neurotoxic effects, reproductive health concerns and endocrine disruption. Depending on their method of action, certain pesticides, such as those determined to be xenoestrogens (organochlorines, organophosphates, and carbamates), will have increased effects based on the developmental stage of individuals when exposure occurs [10]. The effects of pesticides in general are felt more readily by certain populations, and for the general population dietary intake is considered to be a major route of exposure [11].

The use of pesticides is pervasive in the agricultural system and due to their success in increasing crop yields it is unlikely that their use will be completely eliminated. Studies summarized here have examined the levels of pesticide residues in various food commodities and attempted to determine the health risk associated with their consumption. Studies of this nature are needed to identify improper use of pesticides, to inform consumers of their potential exposures, 
determine the health risks associated with exposures, and to identify steps that can be taken to mitigate exposures.

\section{Monitoring of pesticides in foods:}

Monitoring pesticide residues is the only way to effectively control the concentrations of pesticides on foods. Recently more programs have been established to monitor pesticides, with surveillance focusing on proper use of pesticides with regards to application rates and compliance with MRLs [1].

Allowable residue limits for a given pesticide vary between food products and the country where they are used [11-13]. Different countries have different standards to which their pesticide residues are held, and have different methods for monitoring their presence. Certain pesticides, although known to be harmful when consumed, do not have maximum residue levels in certain countries [12].

In the United States (US) the US Department of Agriculture's (USDA) Agricultural Marketing Service (AMS) has been regularly monitoring the U.S food supply for pesticide residues since May of 1991 through the Pesticide Data Program (PDP) [14]. Each year the USDA and Environmental Protection Agency (EPA) collaboratively decide which foods need to be tested on a rotating basis. The latest report, released in May of 2012, includes data from 2010 during which time fresh and processed fruit and vegetables, oats, eggs, catfish, baby food, groundwater and treated and untreated drinking water were all tested for pesticide residues [14].

The European Union (EU) also has a rigorous system in place for monitoring pesticides, and each year the European Food Safety Authority assesses pesticide residues at pre and post-harvest regulation levels [11]. Existence of monitoring programs like those in the US and EU are not the norm in many other countries, particularly developing countries. In developing countries pesticide control programs are not as rigorously upheld and farmers may take short-cuts in the prescribed pesticide application due to high demand for their crops and the low perception of pesticide toxicity in humans [1]. Some developing countries export a good amount of their fresh produce making pesticide monitoring programs important for both local and non-local consumers. Often times in countries where food is consumed locally and exported, domestic foods are less monitored than exported foods due to restrictions established by importing countries [13]. Exports have to pass strict phytosanitary requirements imposed by importing countries, so resources are used testing exports' pesticide levels instead of domestic crops [13].

Monitoring of pesticide levels needs to be equal across all food commodities regardless of intended distribution location in order to protect consumers. Pesticide residues in foods need to be continuously monitored and MRLs for all licensed pesticides within a country need to be established to ensure their proper use. In one study, detectable levels of residues for pesticides that had been banned from use for a number of years were found in some fruit and vegetable products, indicating that possible misuse of products containing these chemicals 
may be occurring [1]. If there is no continuous monitoring program to ensure proper use of pesticides harmful practices may be developed.

\section{Levels of pesticides found in foods}

Pesticide residues present at time of harvest have been shown to be related to pesticide application times and dosages. It has been shown that food storage, transportation, storage conditions and duration, interlaboratory variations and analytical methods used to measure pesticide residue levels may also affect the determined amounts of residues in food products [1, 2, 8]. Studies summarized here examined a variety of pesticides and their residue levels in a variety of foods in comparison to the associated MRLs. When available, data on actual residue concentrations detected above the established MRLs is provided (Tables 1 and 2).

Table 1: $\quad$ Selection of pesticide levels above their MRL in tested food products.

\begin{tabular}{|c|c|c|c|c|c|}
\hline $\begin{array}{l}\text { Pesticide } \\
\text { analyzed }\end{array}$ & $\begin{array}{l}\text { \# Samples } \\
\text { with } \\
\text { residues }\end{array}$ & $\begin{array}{c}\text { \# Samples } \\
\text { with } \\
\text { residues > } \\
\text { mrl }\end{array}$ & $\begin{array}{c}\mathrm{Mrl} \\
(\mathrm{mg} / \mathrm{kg})\end{array}$ & $\begin{array}{c}\text { Detected } \\
\text { concentration } \\
(\mathrm{mg} / \mathrm{kg})\end{array}$ & Reference \\
\hline Boscalid & 4 & 3 & 0.01 & $0.02-0.20$ & [9] \\
\hline Chlorpyrifos & 93 & 28 & 0.05 & $0.005-1.51$ & [9] \\
\hline Chlorothalonil & 3 & 3 & 0.01 & $0.012-0.28$ & [9] \\
\hline Dimethoate & 3 & 2 & 0.02 & $0.01-0.05$ & [9] \\
\hline Carbaryl & 18 & 17 & $0.05-0.5^{*}$ & $0.07-2.23$ & [8] \\
\hline Lindane & 1 & 1 & 0.01 & 0.34 & [8] \\
\hline Dieldrin & 1 & 1 & $\begin{array}{l}0.01- \\
0.03 *\end{array}$ & 0.45 & [8] \\
\hline Propoxur & 8 & 8 & 0.05 & $0.56-2.0$ & [8] \\
\hline Chlorpyrifos & 1 & 1 & $0.05-1.0^{*}$ & 6.21 & [8] \\
\hline Tefluthrin & 1 & 1 & 0.05 & 0.08 & [8] \\
\hline $\begin{array}{l}\text { Tolclofos- } \\
\text { methyl }\end{array}$ & 7 & 4 & $0.05-2 *$ & $0.24-7.31$ & [8] \\
\hline Metalaxyl & 3 & 2 & $0.05-2 *$ & $0.26-1.05$ & {$[8]$} \\
\hline Paraquat & 3 & 3 & 0.02 & $0.38-1.23$ & [8] \\
\hline Bromoxynil & 1 & 1 & 0.05 & ND-2.29 & [8] \\
\hline Amitrol & 2 & 2 & 0.01 & $0.77-0.87$ & [8] \\
\hline Dicofol & 2 & 1 & $0.02-1^{*}$ & $0.05-0.147$ & [8] \\
\hline Carbofuran & 11 & 11 & 0.02 & $0.19-1.62$ & [8] \\
\hline Permethrin & 16 & 9 & 0.05 & $0.004-0.448$ & [13] \\
\hline Chlorpyrifos & 3 & 1 & 0.05 & $0.01-0.08$ & [13] \\
\hline
\end{tabular}

*In Saudi Arabia there are varying MRLs for this pesticide depending on the food commodity it is isolated from. Samples listed as above the MRL were determined using the established MRL and pesticides detected for a specific food type. 
Table 2: Pesticide/food pairs exceeding the MRL in the United States, 2010 [15].

\begin{tabular}{|l|l|c|c|c|c|}
\hline \multicolumn{1}{|c|}{ Pesticide } & \multicolumn{1}{|c|}{ Food } & $\begin{array}{c}\text { \# Samples \# Samples } \\
\text { with } \\
\text { residues }\end{array}$ & $\begin{array}{c}\text { Detected } \\
\text { with } \\
\text { residues } \\
\text { concentration } \\
\text { (ppm) }\end{array}$ & $\begin{array}{c}\text { MRL } \\
\text { (ppm) }\end{array}$ \\
\hline Acephate & Cucumbers & 5 & 1 & $0.003-0.043$ & 0.02 \\
\hline & $\begin{array}{l}\text { Frozen } \\
\text { spinach }\end{array}$ & 2 & 2 & $0.087-1$ & 0.002 \\
\hline & Watermelon & 3 & 3 & $0.16-0.27$ & 0.02 \\
\hline Chlorfenapry & Cucumbers & 13 & 1 & $0.004-0.022$ & 0.01 \\
\hline Chlorpyrifos & Cilantro & 150 & 2 & $0.002-0.67$ & 0.1 \\
\hline & Cucumbers & 24 & 1 & $0.002-0.45$ & 0.1 \\
\hline Cyhalothrin & Asparagus & 2 & 2 & $0.043-0.08$ & 0.01 \\
\hline & $\begin{array}{l}\text { Frozen } \\
\text { spinach }\end{array}$ & 2 & 2 & $0.042-0.32$ & 0.01 \\
\hline Cypermethrin & Asparagus & 1 & 1 & 0.072 & 0.005 \\
\hline Fludioxonil & Sweet Bell & 2 & 2 & $0.055-0.059$ & 0.01 \\
& Peppers & & & & \\
\hline Imidacloprid & Grapes & 357 & 2 & $0.004-2.3$ & 1 \\
\hline Methidation & Pears & 4 & 1 & $0.005-0.068$ & 0.05 \\
\hline Peperonyl & Sweet & 39 & 7 & $0.015-0.89$ & 0.25 \\
& potatoes & & & & \\
\hline Thiabendazole & Apples & 601 & 1 & $0.002-7.4$ & 5.0 \\
\hline Thiamethoxam & Sweet Bell & 198 & 2 & $0.005-0.27$ & 0.25 \\
& Peppers & & & & \\
\hline & & & & \\
\hline
\end{tabular}

The United States monitoring program focuses on foods commonly consumed by infants and children [14]. Pesticide residues were found to almost always be well below the MRLs established by the EPA. In 2010 there were 12,028 samples tested from contributing states [14]. Forty-one percent of samples tested had no detectable pesticides, $18.5 \%$ had one pesticide, and $40.5 \%$ contained more than one pesticide. There were 30 samples $(0.25 \%)$ with pesticides exceeding MRLs (Table 2), and 17 of them were reported as being imported [15]. Some pesticides were found to exceed the MRL in $100 \%$ of pesticide/food pairs tested (acephate in frozen spinach and watermelon, and others) [15]. Some of the MRLs were noticeably exceeded, and others only slightly: acephate was found to be over the MRL in one cucumber sample, with $0.043 \mathrm{ppm}$ detected $(\mathrm{MRL}=0.02 \mathrm{ppm})$, while Methidation was found at $0.068 \mathrm{ppm}(\mathrm{MRL}=0.05 \mathrm{ppm})$ in one Pear sample [15]. The complete list of pesticide/food samples with limits over the MRL in the US is shown in Table 2. 
Instead of focusing on a vulnerable population like the US, the majority of other studies focused on a specific food type: typically fruits and vegetables, or in one study, wheat. In Belgium residues were found in $72 \%$ of fresh fruits and vegetables, but $94 \%$ of the positive samples were below maximum residue levels [2]. Similar to the United States, imported fruits and vegetables were found to have slightly higher occurrences of residues above the MRL [2].

A study in China found that 353 samples (11.7\%) contained pesticide residues above MRLs, 782 samples (26\%) had pesticide levels at or below the MRL and 1874 samples (62.3\%) had no detectable pesticide residue [1]. Certain food groups were found to be more problematic than others, with $17.2 \%$ of pakchoi cabbages having levels above the MRL while no pesticides were detected on any oranges or apples [1]. The most commonly detected pesticide over the MRL was methamidophos, with $5.5 \%$ of positive samples above the MRL [1]. The lowest detected pesticides were Cyhalothrin, Dichlorvos, Fenpropathrin, and Fenvalerate which were found over the MRL in $0.1 \%$ of positive samples. [1]

Unlike China where certain foods were more likely to have pesticides, in Poland individual pesticides were found to be more common [9]. The recent study conducted on Brassica vegetables found that Chlorpyrifos and Cypermethrin were more common than others, and were found in $27.4 \%$ and $3.3 \%$ of samples respectively [9]. Lower percentages of pesticides above the MRL were found in Poland compared to China, with only thirty three samples (9\%) exceeding the MRL [9]. Some of the samples that exceeded the MRL in Poland were only slightly over the MRL (Chlorothalonil found at $0.012 \mathrm{mg} / \mathrm{kg}$, MRL $=0.01$ ), while others were more noticeably over the MRL, as shown in Table 1 [9].

In Saudi Arabia it was found that $56 \%$ of samples had pesticide residues and $34 \%$ of samples had levels above the MRL [8]. Some pesticides were found to exceed MRL by a substantial amount (lindane found at $0.34 \mathrm{mg} / \mathrm{kg}, \mathrm{MRL}=0.01$ $\mathrm{mg} / \mathrm{kg}$ ) and others were found to be closer to the MRL (tefluthrin detected at $0.05 \mathrm{mg} / \mathrm{kg}, \mathrm{MRL}=0.08 \mathrm{mg} / \mathrm{kg}$ ) (see Table 1) [8]. The number of pesticides present at or above MRLs can't be completely determined in this study however, as 7 of the 23 pesticides tested have no established MRLs [8].

A study done in Korea found that of the seven pesticides licensed for use in Yuza production only 3 were found in detectable levels on the fruit, and all were below their respective MRLs [16]. A three year study on fresh fruits and vegetables in Croatia found 247 (28.5\%) of samples had pesticide residues at or below the established MRL and 46 (5.3\%) contained pesticides above the MRL [3].

The wheat industry in South Africa is one of the major users of pesticides, and pesticides were detected in all local in imported samples [13]. Eight pesticides were detected in total, with mercaptothion being the most frequently detected, followed by permethrin and chlorpyrifos [13]. Nine of the samples exceeded the MRL for permethrin $(0.05 \mathrm{mg} / \mathrm{kg})$, with 7 of them being local samples and 2 imported. One imported sample had chlorpyrifos above the MRL (0.08 mg/kg, MRL =0.05 mg/kg), as seen in Table 1 [13]. 
Studies typically tested multiple pesticides in different food products. The levels of pesticides found in a given food product ranged from $100 \%$ of local wheat samples in South Africa containing Mercaptothion [13], to $0.013 \%$ of fruits, vegetables and baby foods containing Allethrin in the United States [15]. There were also certain food types in different studies that were found to have no residues from certain types of pesticides; these samples were not included in the table.

The presence of multiple pesticides should be considered as well, and in Xiamen, China it was found that 440 (14.6\%) fruit and vegetable samples tested had residues from two or more different pesticides, with some containing as many as four [1], The potential reduction in residues from processing, and health effects caused by multiple pesticide residues' needs to be considered.

\section{Exposure to pesticide residues in food}

Once the presence of pesticide residues on food have been determined, actual exposure to the chemicals though ingestion or physical contact needs to be determined in order to assess potential health risk. Levels of pesticides on foods are typically monitored with respect to their MRL, while pesticide levels associated with health risks are measured in ADI and ARfD and take consumption rates into consideration. Since MRLs are based on good agricultural practices and ADI/ARfDs are based on toxicological data, it is possible for foods to have pesticide levels above the MRLs that do not pose any health risk.

Determining exposure values based on pesticide residue levels and food consumption can be done using a deterministic or probabilistic approach. The deterministic approach is simpler and based on single point estimates for each variable in the model [2]. The probabilistic approach allows for all possible values for each variable to be taken into account and each possible model outcome is weighted by the probability of its occurrence [2]. This is advantageous in that all available data are used, the exposure estimate is presented as a distribution, and variability and uncertainty can be quantified [2]. The deterministic approach may be used as a screening tool to identify problematic pesticides, followed by the probabilistic approach to see if the point estimate actually gives rise to concerns [2].

When performing risk assessments it is also important to consider changes in pesticide residues that may occur during industrial and household processes [3, 12]. Residue levels are often prior to processing and residue concentrations can increase or decrease during processing depending on the properties of the residue and conditions of processing. Uncertainty factors can be applied when modeling exposure risk to account for changes after processing, but when available they are only approximations [2]. None of the studies summarized here used pesticide levels post-processing. Current pesticide residue statuses were not concluded to be of imminent public health importance in any of the studies, with the exception of some high risk populations when high levels of consumption were possible. 
The most recent reports in the United States indicate that overall pesticide residues on foods are not a health risk [15]. In Belgium, it was found that certain vulnerable populations (children) had consumption levels for imazalil and Prochloraz that significantly exceeded the ADI $(0.025 \mathrm{mg} / \mathrm{kg}$ bw day) when fruits and vegetables were consumed frequently or in large amounts [2]. This risk assessment used consumption data from German children however, and therefore may not accurately represent health risk in Belgium children [2]. In the general population of Belgium the chronic intake of the 29 considered pesticide residues was low compared to the ADI, with most of them being less than $1 \%$ of the ADI [2].

In the studies conducted in Poland, China, South Africa, Korea and Saudi Arabia it was determined that the dietary intakes of pesticide residue couldn't currently be considered a public health issue. Potential health risks from pesticide residues in Poland were estimated using pesticide residue detection and consumption data. Of the 93 samples that tested positive for Chlorpyrifos, 23 were above the MRL, but the combined cumulative exposures for Chlorpyrifos in Brassica were $0.777 \%$ of the acceptable daily intake [9]. (Table 3) The estimated daily intake for pesticides ranged from $0.005 \%$ to $4.454 \%$ of the ADI when high consumption rates were assumed [9]. Comparisons between residue levels and actual exposure based on high and average consumption patterns are shown in Table 3 [9].

Table 3: Estimates of actual exposure to pesticides through Broccoli consumption [9].

\begin{tabular}{|l|c|c|c|c|c|}
\hline \multicolumn{1}{|c|}{ Pesticide } & $\begin{array}{c}\text { Average } \\
\text { residue } \\
\text { levels } \\
\text { (mg/kg) }\end{array}$ & $\begin{array}{c}\text { MRL } \\
\text { (mg/kg) }\end{array}$ & $\begin{array}{c}\text { Acceptable } \\
\text { daily intake } \\
\text { (mg/kg } \\
\text { b.w.) }\end{array}$ & $\begin{array}{c}\text { British adults } \\
\text { (76k) \%ADI- } \\
\text { high } \\
\text { consumption }\end{array}$ & $\begin{array}{c}\text { British adults } \\
\text { (76 kg) \%ADI } \\
\text { Avg } \\
\text { consumption }\end{array}$ \\
\hline Chlorpyrifos & 0.4005 & 0.05 & 0.01 & 0.357 & 0.03 \\
\hline Diazinon & 0.01 & 0.02 & 0.0002 & 4.454 & 0.375 \\
\hline Dimethoate & 0.01024 & 0.02 & 0.001 & 0.912 & 0.077 \\
\hline Fenitrothion & 0.0201 & 0.5 & 0.005 & 0.358 & 0.03 \\
\hline a-cypermethrin & 0.02015 & 0.5 & 0.015 & 0.12 & 0.01 \\
\hline Bifenthrin & 0.03 & 0.2 & 0.015 & 0.178 & 0.015 \\
\hline Cypermethrin & 0.02155 & 0.5 & 0.05 & 0.038 & 0.003 \\
\hline Esfenvalerate & 0.02 & 0.04 & 0.02 & 0.089 & 0.008 \\
\hline Fenvalerate & 0.02 & 0.04 & 0.0125 & 0.143 & 0.012 \\
\hline Indoxacarb & 0.0502 & 0.3 & 0.006 & 0.745 & 0.063 \\
\hline$\lambda$-cyhalothrin & 0.02 & 0.1 & 0.005 & 0.356 & 0.03 \\
\hline Azoxystrobin & 0.04 & 0.5 & 0.2 & 0.018 & 0.002 \\
\hline Boscalid & 0.01126 & 0.01 & 0.04 & 0.025 & 0.002 \\
\hline Chlorothalonil & 0.01105 & 0.01 & 0.015 & 0.066 & 0.006 \\
\hline Pyrimethanil & 0.01005 & 0.5 & 0.17 & 0.005 & 0.0004 \\
\hline & & & & &
\end{tabular}


In China dietary intakes do not appear to pose a health risk to local consumers, but the intake estimated from the highest pesticide levels is close to or above short-term health standards [1]. In South Africa, WHO cluster diets were used to determine consumption, and despite being present in nearly all samples, the average and highest levels of mercaptothion resulted in intakes below $1 \%$ of the ADI [13]. In the Korean study, a ratio of estimated daily intake to acceptable daily intake of pesticides was used to determine that the current pesticide levels were not harmful to human health [16]. Although there were pesticide residues found on more than half of the samples tested in Saudi Arabia, and almost a third with levels above the MRLs, the intake of pesticides was found to be lower than the ADI, below the level to produce health risk [8].

In order to establish a worst case scenario for acute exposure, the authors of the Croatian study decided to combine the highest consumption levels with the highest residues measured in their monitoring programs [3]. When this was done, a potential consumer risk was found for short term exposure to 8 pesticide/food combinations in children ( $>150 \%$ of ARfD) and for 3 pesticide/food combinations in adults ( $>150 \%$ ARfD) [3]. Long-term risk assessments were done comparing the calculated exposure with the mean pesticide residue levels consumed and the ADI. The long-term exposure was determined to be low for Croatians, and similar results were found in the EU in other studies [3]. In most cases the exposure accounted for less than $25 \%$ of the ADI [3].

Many studies examine the risk of exposure to a single pesticide residue from a single food product based on that food product's consumption, but people are exposed to multiple pesticides simultaneously. There is currently no internationally accepted procedure for evaluating cumulative exposures to multiple pesticides and risk assessment of pesticide residues in food is based on toxicological evaluation of the single compounds [3]. In order to fully understand the risks associated with pesticide consumption the effects of multiple pesticides needs to be evaluated.

\section{Fumigants used with foods:}

Fumigants, typically applied directly to food products post-harvest, are insecticides in a gaseous state that can spread through all areas of sealed structures and/or areas [17]. They are highly toxic and can access small spaces, making them invaluable in the treatment of stored food products [17]. The characteristics that make fumigants highly useful also make them dangerous. If containers used for fumigating food products are not properly sealed fumigants make leak and harm animals or people [17]. Like other pesticides, fumigants can potentially leave residues on foods and their consumption can be harmful to human health [18].

The EPA does require monitoring of fumigant pesticides, but the requirements only specify testing of ambient air concentrations where fumigation occurs and in the near vicinity [19]. There are established chronic reference doses for the amount of phosphide fumigants that can be consumed daily without 
causing adverse effects $(0.0113 \mathrm{mg} / \mathrm{kg} /$ day) [19], and it has been stated that improper waiting times between fumigation with phosphides and handling could result in exposures above MRLs [20]. When food products are fumigated with aluminum phosphide the food products must be aerated for a minimum of 48 hours prior to their contact with consumers, tobacco products must be aerated for 2-3 days depending on the fumigation structure [21]. Few studies have been conducted to examine the actual levels of fumigants on foods when they're distributed to consumers.

Fumigants used directly on stored food products to decrease levels of pests in foods are very beneficial in protecting crops. Additional studies testing the levels of actual pesticide residues on fumigated food products could be beneficial in determining exposure to fumigants through food consumption.

\section{Conclusions}

Pesticides are commonly used in agriculture to increase crop yields and the health risks associated with their residues on food need to be continuously evaluated. ADIs and ARfDs need to be used rather than MRLs when calculating health risk. Even though some studies in this review did not find pesticide levels to be a current health risk, the fact that levels are above MRLs indicates improper use and reinforces the need for continuous monitoring. Conducting health risk assessments requires a number of assumptions, so keeping pesticide residues to a minimum is important in protecting consumer health.

Despite their direct application to food products, fumigant residues have not been extensively studied in recent literature. The determinations of fumigant residues, effects of multiple residues, and residue levels after processing are all important topics for future studies to protect consumers.

\section{References}

[1] Chen Chen, Y.Q., Qiong Chen, Chuanjiang Tao, Chuanyong Li, Yun Li, Evaluation of pesticide residues in fruits and vegetables from Xiamen, China. Food Control, 2011, 22 (2011): p. 1114-1120.

[2] Wendie L Claeys, J.-F.S., Claude Bragard, Guy Maghuin-Rogister, Exposure of several Belgian consumer groups to pesticide residues through fresh fruit and vegetable consumption. Food Control, 2010, 22 (2011): p. 508-516.

[3] Zorka Knezevic, M.S., Marijan Ahel, Risk assessment of the intake of pesticides in Croatian diet. Food Control, 2012, 23: p. 59-65.

[4] Nida Besbelli, B.E., Ruth Etzel, Pesticides, WHO, Editor 2008, WHO.

[5] Types of Pesticides. 2012 5/9/12 [cited 2013; Available from: http://www.epa.gov/pesticides/about/types.htm\#type.

[6] Frequently asked questions about Organophosphates, D.o.H.a.H. Services, Editor 2013, Centers for Disease Control and Prevention.

[7] Pyrethroids and Pyrethrings, U. EPA, Editor October 2012, Environmental Protection Agency. 
[8] Khalde A. Osman, A.I.A.-H., A.M. Al-Rehiayani, K.N. Al-Redhaiman, Estimated Daily intake of pesticide residues exposure by vegetables grown in greenhouses in Al-Qassim region, Saudi Arabia. Food Control, 2011, 22: p. 947-953.

[9] B Lozowicka, M.J., R Kazcynski, Pesticide residues in Brassica vegetables and exposure assessment of consumers. Food Control, 2011, 25: p. 561575.

[10] S. Inigo-Nunez, M.A.H., T. Encinas, A Gonzalez-Bulnes, Estimated daily intake of pesticides and xenoestrogenic exposure by fruit consumption in the female population ofrom a Mediterranean country (Spain). Food Control, 2010. 21: p. 471-477.

[11] Alexandre Nougadere, V.S., Ali Kadar, Antony Fastier, Eric Truchot, Claude Vergnet, Frederic Hommet, Joelle Bayle, Philippe Gros, JeanCharles Leblanc, Total diet study on pesticide residues in France: Levels in food as consumed and chronic dietary risk to consumers. Environmental International, 2012. 45: p. 135-150.

[12] Zhang Cun-zheng, Z.X.-M., Tian Zi-Hua, He Dan-Jun, Liu Xian-Jin, Degredation of Chlorpyrifos and Fipronil in Rice from Farm to Fork and Risk Assessment. Science Direct, 2010. 9(5): p. 754-763.

[13] Mohamed Aqiel Dalvie, L.L., Risk assessment of pesticide residues in South African raw wheat. Crop Protection, 2009. 28: p. 864-869.

[14] Michael T Jarvis, D.K., Shelly L Burgess, USDA Releases 2010 Annual Summary for Pesticide Data Program Report, confirms that U.S., U.S.D.o. Agriculture, Editor 2012, Agriculture Marketing Service.

[15] Shipman, D., Pesticide Data Program, Annual Summary, Calendar Year 2010, U.S.D.o. Agriculture, Editor 2012, USDA.

[16] Kwang-Geun Lee, S.-K.L., Monitoring and risk assessment of pesticide residues in yuza fruits (Citrus junos Sieb. ex Tanaka) and yuza tea samples produced in Korea. Food Chemistry, 2012. 135: p. 2930-2933.

[17] Nathan J. Dyrud, D.H., Private Stored Grain Fumigation Training Manual, 2001, University of Minnesota Extension Service.

[18] Fishbein, L., Potential Hazards of Fumigant Residues. Environmental Health Perspectives, 1976, 14: p. 39-44.

[19] Aluminum and Magnesium Phosphide, U.S.E.P. Agency, Editor 1998, EPA R.E.D. Facts Prevention, Pesticides and Toxic Substances.

[20] Bullen, K., Grain storage - short cuts for insect control, in DPI\&F Note 2005, State of Queensland Department of Primary Industries and Fisheries.

[21] Applicator's Manual for Aluminum Phosphide Fumigant Tablets, Pellets and Gas Bags, 2010, United Phosphorus Inc. 